Supplement of

\title{
Identification of snowfall microphysical processes from Eulerian vertical gradients of polarimetric radar variables
}

Noémie Planat et al.

Correspondence to: Alexis Berne (alexis.berne@epfl.ch)

The copyright of individual parts of the supplement might differ from the article licence. 

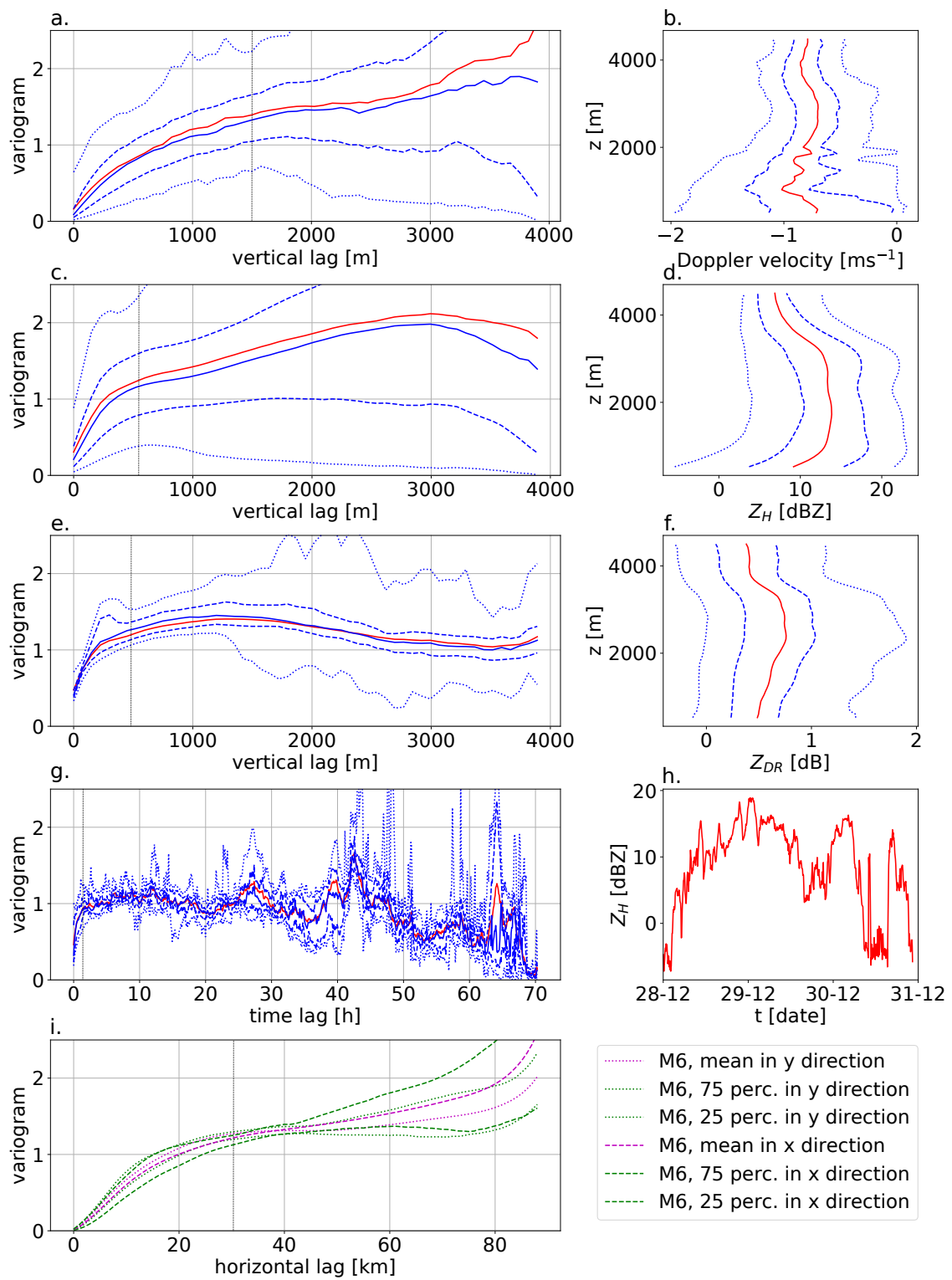

M6, mean in y direction

M6, 75 perc. in y direction

M6, 25 perc. in y direction

M6, mean in $x$ direction

M6, 75 perc. in $x$ direction

M6, 25 perc. in $x$ direction

Figure 1. Variogram analysis during EV1. (a) vertical empirical variogram of the Doppler velocity along the vertical direction (statistics refer to the temporal dimension). (b) vertical profiles of the Doppler velocity averaged in time. Vertical experimental variograms for $Z_{H}$ (c) and $Z_{D R}$ (e). Vertical profiles of $Z_{H}$ (d) and $Z_{D R}$ (f) averaged in time and horizontally. (g) temporal empirical variogram of $Z_{H}$. (h) spatially averaged $Z_{H}$ as a function of time. In panels (a) to (h), red lines show the mean profiles, solid blue lines the median profiles, and dashed (resp. dotted) lines the 25-th and 75-th percentiles (resp the 5-th and 95-th percentiles). The vertical black dotted lines in (a), (c), (e), (g) correspond to the characteristic scales measured and reported in Table 1. (i) in dashed (resp. dotted) magenta line, the horizontal variogram in $x$ direction (resp. $y$ direction) of the sixth moment of the snow particle size distribution during all the event from the WRF simulation (see Appendix A). In dashed green (resp .dotted green) line the 25-th and 75-th percentiles in the $x$ (resp. $y$ ) direction. $x$ and $y$ designate model axes (shifted with respect to longitude/latitude, see Vignon et al. (2019)). 

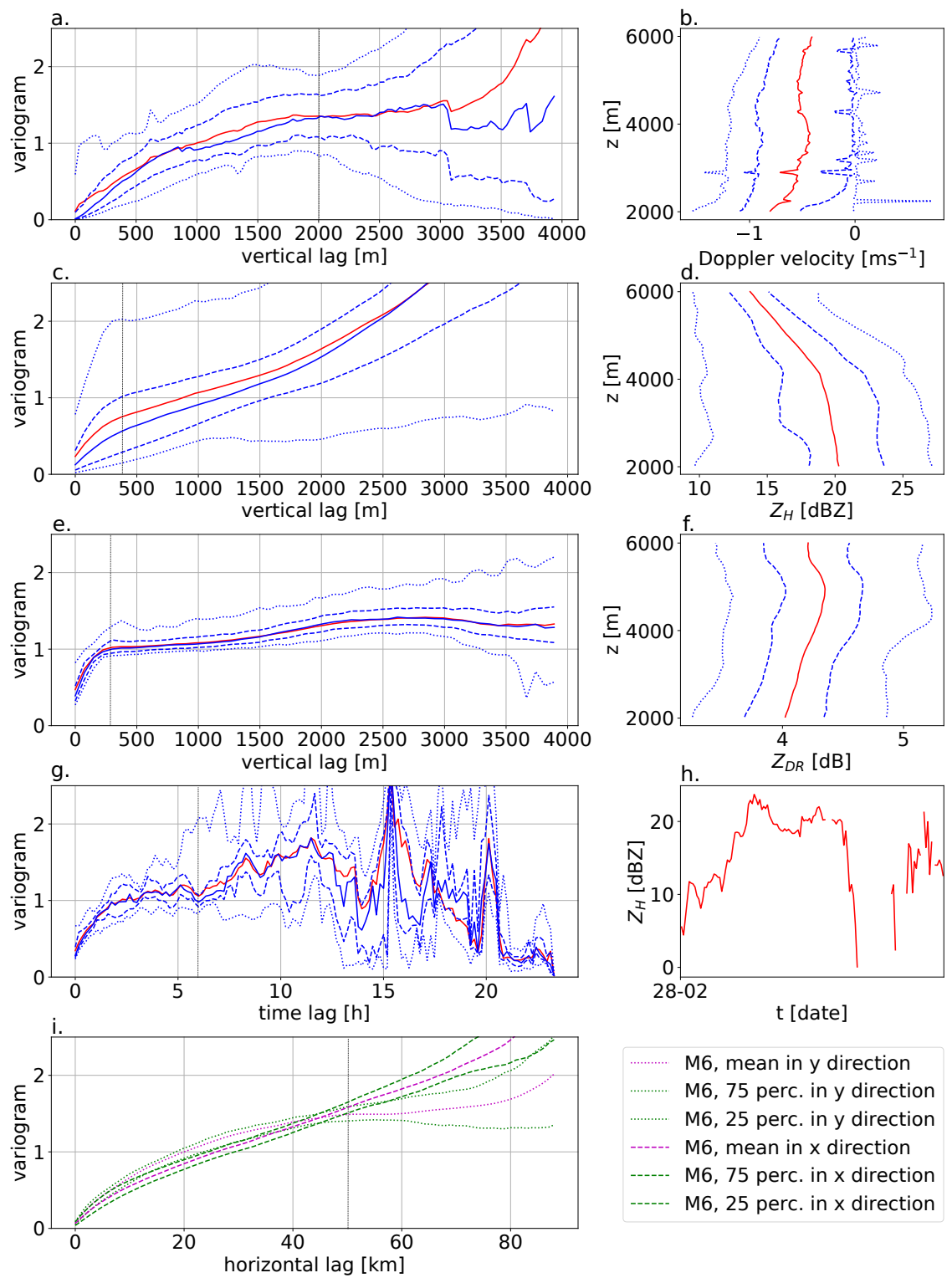

M6, mean in y direction

M6, 75 perc. in y direction M6, 25 perc. in y direction

M6, mean in $x$ direction

M6, 75 perc. in $x$ direction

M6, 25 perc. in $x$ direction

Figure 2. Same as Fig. 2 for EV2

\begin{tabular}{|c||c|c|}
\hline Parameter & EV1 & EV2 \\
\hline$p[\%]$ & 25 & 25 \\
\hline$D_{x}[\mathrm{~km}]$ & 10 & 15 \\
\hline$D_{z}[\mathrm{~km}]$ & 4.5 & 6 \\
\hline$\Delta t[\mathrm{~min}]$ & 10 & 10 \\
\hline$\Delta x[\mathrm{~km}]$ & 1 & 1 \\
\hline
\end{tabular}

Table 1. Numerical values of the processing parameters of the PIVS method for each of the two case studies. 


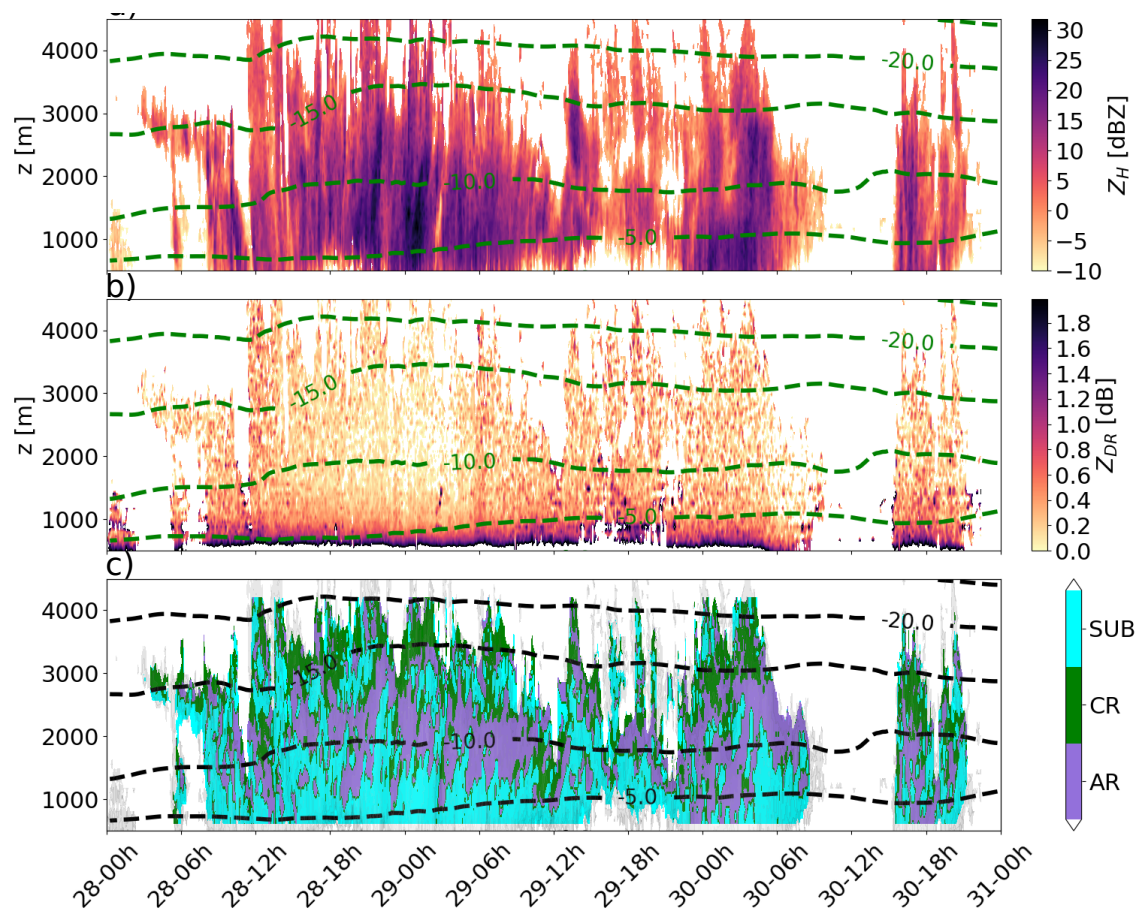

Figure 3. Height-time plot of $Z_{H}$ (a) and $Z_{D R}$ (b) for EV1, together with the dominant process type identified with the PIVS method (c). Dashed green (a,b) and black (c) lines show the temperature obtained from the WRF numerical simulation (see Appendix A of the main manuscript for details) and extracted along the same RHI as the radar. For measured temperature and RHi, please refer to the Radiosoundings visible on Fig. 3 of the paper 


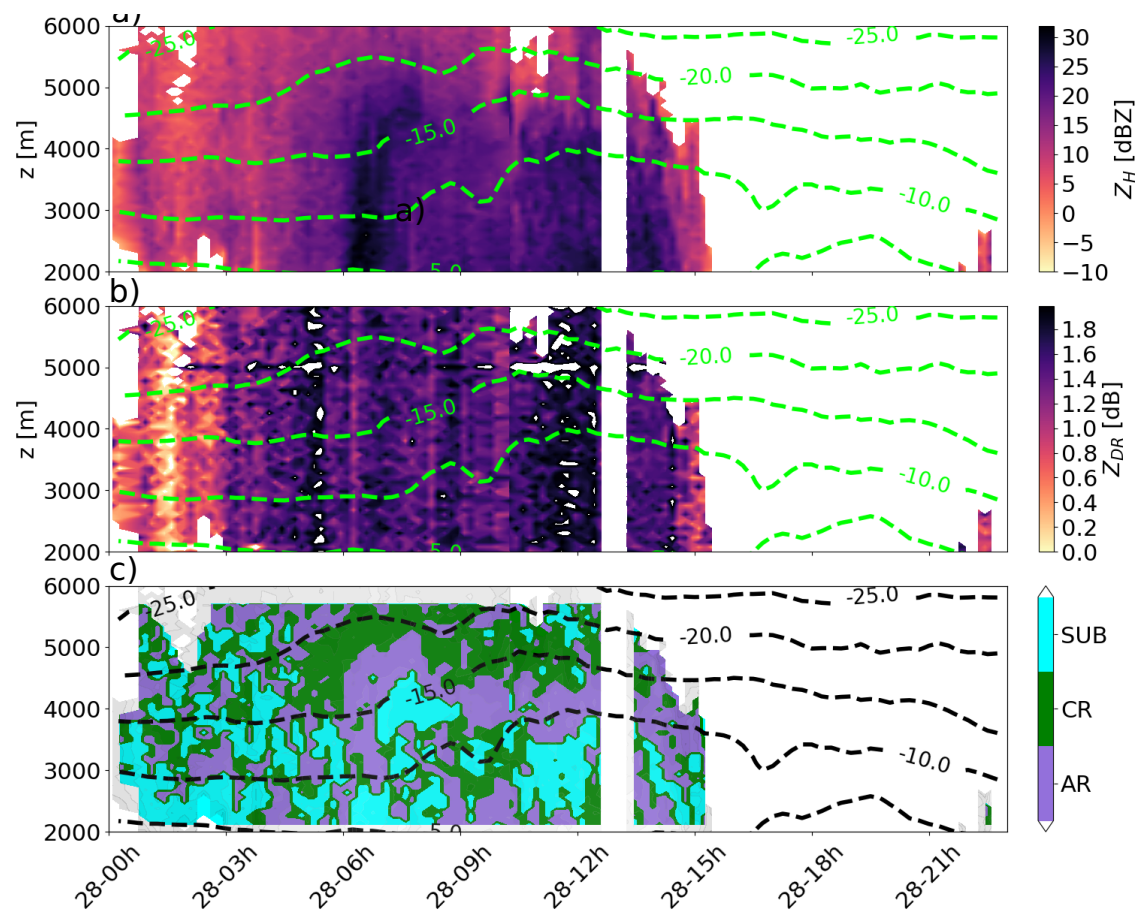

Figure 4. Same as Fig. 3 for EV2 


\section{References}

Vignon, E., Besic, N., Jullien, N., Gehring, J., and Berne, A.: Microphysics of Snowfall Over Coastal East Antarctica Simulated by Polar WRF and Observed by Radar, Journal of Geophysical Research: Atmospheres, 124, https://doi.org/10.1029/2019JD031028, 2019. 